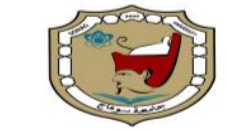

Sohag University

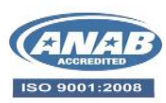

Sohag Medical Journal

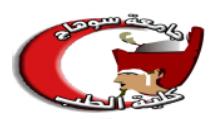

Faculty of Medicine

\title{
The Impact of Programmed Death-1 on Immune Thrombocytopenia
}

\author{
Hasnaa Ahmed Aboalwafa, Doaa Mohamed Bardis, Heba Abdul Hafez \\ Ahmed, Hesham M. Hefney, Ahmed Ahmed Allam. \\ clinical and chemical pathology faculty of medicine Sohag university
}

\begin{abstract}
:
Background programmed death (PD-1) has an important role in inhibiting the immune system in immune thrombocytopenic purpura (ITP) by switching off auto-reactive T-cells. This work aims to assess the expression of (PD)-1 negative co-stimulatory molecule in patients with ITP and to detect its relation with platelet count. Patient and methods, forty patients were newly diagnosed as ITP 13 males and 27 females were included, their age ranged from 1 to 43 years and twenty-six healthy subjects 8 males and 18 females as control group their age ranged from 5 to 62 year. Expression of PD-1+CD4+ T-cells by B.D FACS Calibur flow cytometry was performed in ITP patients and healthy subjects on peripheral blood samples, Mo Abs supplied by BD Bioscience, United States. PD-1 FITC Lot: GR 145543-6 and CD4 PE Cat: 555347, San Jose, California. Negative mouse isotypic control (PE Lot: 0279236, FITC Lot: 0273533). Results, the percentage of expression of PD-1 on CD4+T cells in the blood from patients with ITP was considerably more than that from healthy subjects $(\mathrm{p}<0.001)$, it has no predictive risk for thrombocytopenia Odd's ratio ( 3.773 ) p ( 0.187), CI ( 0. 525 - 27.09). Conclusion: Increased expression of PD-1 molecule on CD-4 T-cells as inhibitory signals to the activated immune system against self-antigens an important part of ITP pathogenesis. Has no predictive role in the degree of thrombocytopenia.
\end{abstract}

Keywords: ITP, PD-1, CD4, Immune, Inhibition.

\section{Introduction:}

Immune thrombocytopenic purpura (ITP) is a common disease in which reduced platelet count $\left(<100 \times 10^{9} / \mathrm{L}\right)$ in the peripheral blood and start by a process of cellmediated and humoral-mediated destruction of own platelets and is presented by various degrees of severity and forms of bleeding, ranging from minor bleeding in the skin and mucosa to more serious, lifethreatening bleeding in the gastrointestinal or cerebral regions ${ }^{(1)}$. Also, patients lose immune tolerance to self-antig- ens on the platelet surface ${ }^{(2)}$. ITP patients have an enhanced activation of Th1 and Th17 cells, as well as enhanced cytokine release by these cells. (3).

PD-1, a member of the CD28/B7 family, is a key immune system inhibitory protein that is found on the stimulated T cells, B cells, dendritic cells (DCS), monocytes, NK cells, and T-regulatory cells. Programmed death ligand-1 (PD-L1) and programmed death ligand-2 (PD-L2) are two ligands for PD-1 (PD-L2). PD-L1 pres- 
ents on the stimulated Langerhans cells, DCs, endothelial cells, and islet cells, and it inhibits inflammation and prevents autoimmune disorders, whereas PD-L2 is found mostly on DCs, macrophages, and certain tumor cells ${ }^{(4)}$. Furthermore, in rheumatoid arthritis patients, activation of the PD-1/PD-L1 signaling pathway significantly reduces the proliferation of Th1 and Th17 cells, as well as the release of IFN- and IL-17 cytokines ${ }^{(5)}$. TFH cells may play an essential role in controlling platelet antibody production in individuals with ITP. ITP patients had a considerably larger proportion of circulating CXCR5+CD4+TFH cells with PD-1high expression than healthy controls. (6ans7). Furthermore, ITP patients had significantly greater frequencies of circulating CXCR5+ CD4+TFH cells with PD-1 high expression, as well as significantly higher serum IL-21 and IL-6 levels ${ }^{(8)}$. In ITP there was an alteration in PD- 1 and PD-L1, as a reduction in SPD-1 and sPDL1 levels. So, there was a positive correlation between sPD-1 levels and platelet counts. As a result, it appears that different levels of PD-1 and PD-L1 may play a role in the pathogenesis of ITP ${ }^{(9)}$.

\section{Study design:}

\section{Subjects:}

The study involved a group of 40 patients newly diagnosed as ITP. 27 female patients $(67.5 \%)$ and 13 male patients $(32.5 \%)$, aged from 1 to 43 years old, and 26 healthy subjects, 6 males, 18 females as controls were enrolled in the study too, their age ranged from 5 to 62 year, they don't have any hematologic or autoimmune illnesses, and they've never had blood transfusions. Between June 2018 and November 2020, patients were recruited from the Hematology Clinic. Inclusion criteria: Only patients with a bleeding tendency who were diagnosed with ITP using the American Society of Hematology's 2011 evidence-based practice guidelines for immune thrombocytopenia were involved in the study. Criteria for exclusion: Patients who had a splenectomy, secondary immune thrombocytopenia, SLE, HCV, HIV, and patients who received steroid therapy previously or drugs for immune suppression. Patients with lymphoma, other causes of secondary thrombocyte-opened, and have a history of other hematologic disorders.

\section{The following were done to all research participants:}

Complete history, with a focus on age, sex as well as onset and duration of the disease: Patients presented with acute onset of the disease. Types of bleeding: skin eruption, ecchymosis, and epistaxis.

Clinical examination with special emphasis on disease presentation, organomegaly, and lymph node examination. Bleeding manifestations were grouped into mucosal bleeding, mucocutaneous, and organ bleeding.

Ethical consideration: Informed written consent was obtained from the patients. The Research Ethics Committee of our University's Faculty of Medicine accepted the study design.

\section{Sampling and laboratory investiga- tions:}

Three milliliters of peripheral venous blood from all patients and controls were collected into K-EDTA tubes (BD) and submitted to the following tests:

1 Cell-Dyne 3700 automatic cell counter for complete blood count (CBC) (Abbott diagnostic, Dallas, USA).

2 Data were acquired and processed using Cell Quest software for immuneophenotyping (IPT) for PD-1 CD4 expressions on a fluorescence-activated cell 
sorter Calibur flow cytometry (Becton Dickinson Biosciences).

Flow cytometry (FCM) assessment: Detection of PD-1+CD4+T-cell, antihuman monoclonal antibodies, and appropriate mouse isotypic antibodies as a control were used, fluoroisothiocyanate (FITC)-conjugated PD-1 and phycoerythrin (PE)-conjugated CD4 (Mo Abs supplied by BD Bioscience, United states, PD-1 (FITC) Lot: GR 145543-6, CD4 (PE) Cat: 555347, San Jose, California ). Negative mouse isotypic control (appropriately labeled according to the Mo Abs used) for determining the non-specific binding of Mo Abs (Lot: 0279236 PE, Lot: 0273533 FITC).

100 microliters of peripheral blood sample were incubated for 15 minutes at roo$\mathrm{m}$ temperature in the dark with 10 liters of PD-1 and CD4 Mo Abs, as directed by the manufacturer. After incubation, red blood cells (RBC) were extracted by simple lysis and rinsed in phosphate-buffered saline (PBS), which also served as a buffer for suspension. An isotypic-matched negative control was employed for each sample in the FCM analysis. The lymphocyte population was defined using a forward and side scatter histogram.

\section{Data analysis}

IBM SPSS Statistics for Windows version 26 was used to examine the data. For quantitative non-parametric values, median and percentiles were used, and for categorized data, both number and percentage were used. Wilcoxon Rank Sum test for non-parametric data comparison between two independent groups. For non-parametric data, a ranked Spearman correlation test was used to investigate the probable relationship between every two variables within each group. The Chi-square test is used to investigate the relationship between two variables or to compare two independent groups in terms of categorized data. The area under the ROC curve value with a $95 \%$ confidence interval was determined to find the PD-1 optimal cut point in predicting ITP using a receiver operating characteristic (ROC) curve. Sensitivity, specificity, positive predictive value, and negative predictive value were assessed after determining the best cut point. In all tests employed in the study, a value of significance of $5 \%$ was adopted.

\section{Results:}

Table 1 and Fig 1,2 provide the demographic and laboratory characteristics of the study groups. Forty ITP patients were included in the research., 27 female patients $(67.5 \%)$ and 13 male patients $(32.5 \%)$, aged from 1 to 43 years old, and 26 healthy subjects, 6 males, 18 females as controls were enrolled in the study too, their age ranged from 5 to 62 year. The PD-1 expression on CD4+Tcell was significantly increased in patients with ITP compared to healthy individuals $(\mathrm{p}<$ 0.001).). Patients with ITP presented with a wide variety of clinical symptoms ranging from mucosal to mucocutaneous bleeding as shown in Table 2. There is a significant positive correlation between PD-1 expression on CD4+T-cell and PLT count. No significant correlation was noted between the expression of PD-1on $\mathrm{CD} 4+\mathrm{T}$-cell and other parameters (pvalue > 0.05) as in Table ${ }^{(3)}$. PD-1 expression significantly decreases in cases presented with mucosal bleeding than cases presented with cutaneous bleeding ( $\mathrm{p}$ $<0.05$ ) as in table ${ }^{(6)}$, ROC curve of PD1 for discriminating cases with mucocutaneous bleeding than those with cutaneous bleeding was presented in Fig ${ }^{(4)}$, the diagnostic performance of PD1 for discriminating cases presented with mucocutaneous bleeding than cases presented with cutaneous bleeding with an area 
under the curve of 0.603 , and a P-value of 0.215. Also, from the analysis of the curve, it can be calculated that the most relevant cut point is $22.55 \%$, with a sensitivity of $50 \%$ and a specificity of $71.9 \%$.

A ROC curve study was performed to determine the optimal PD-1 cutoff level for predicting ITP, and it was found that
The Impact of Programmed Death-1 on Immune

Doaa mohamed bardis

the optimal cutoff for PD-1 is 10.27 area under a curve of 0.976998 , with a sensitivity of $97.5 \%$, specificity of 100 $\%$, and P-value of 0.001 . as in Table 4. The predictive value of PD1 for the degree of thrombocytopenia was (Odd's ratio 3.773) with a confidence interval from (0.525 to 27.098) and P-value $(0.187)$ as in table ${ }^{(5)}$

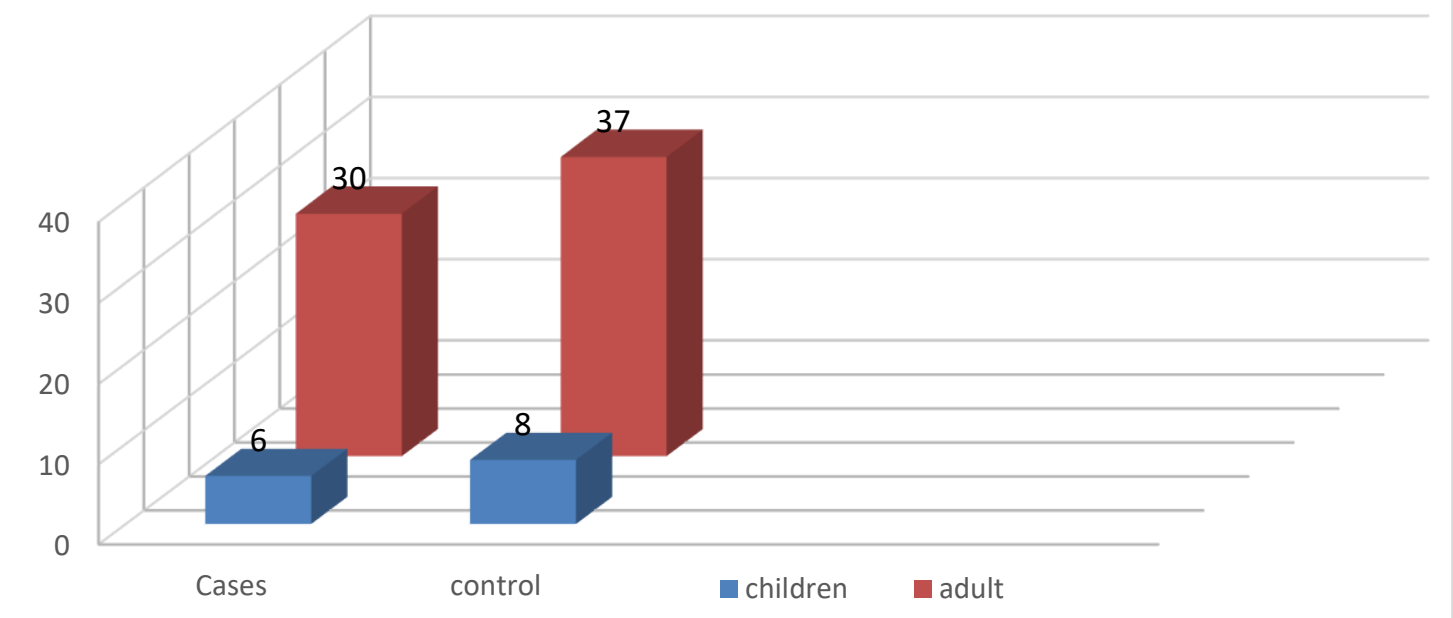

Fig(1a): Median age in adults and children of studied groups.

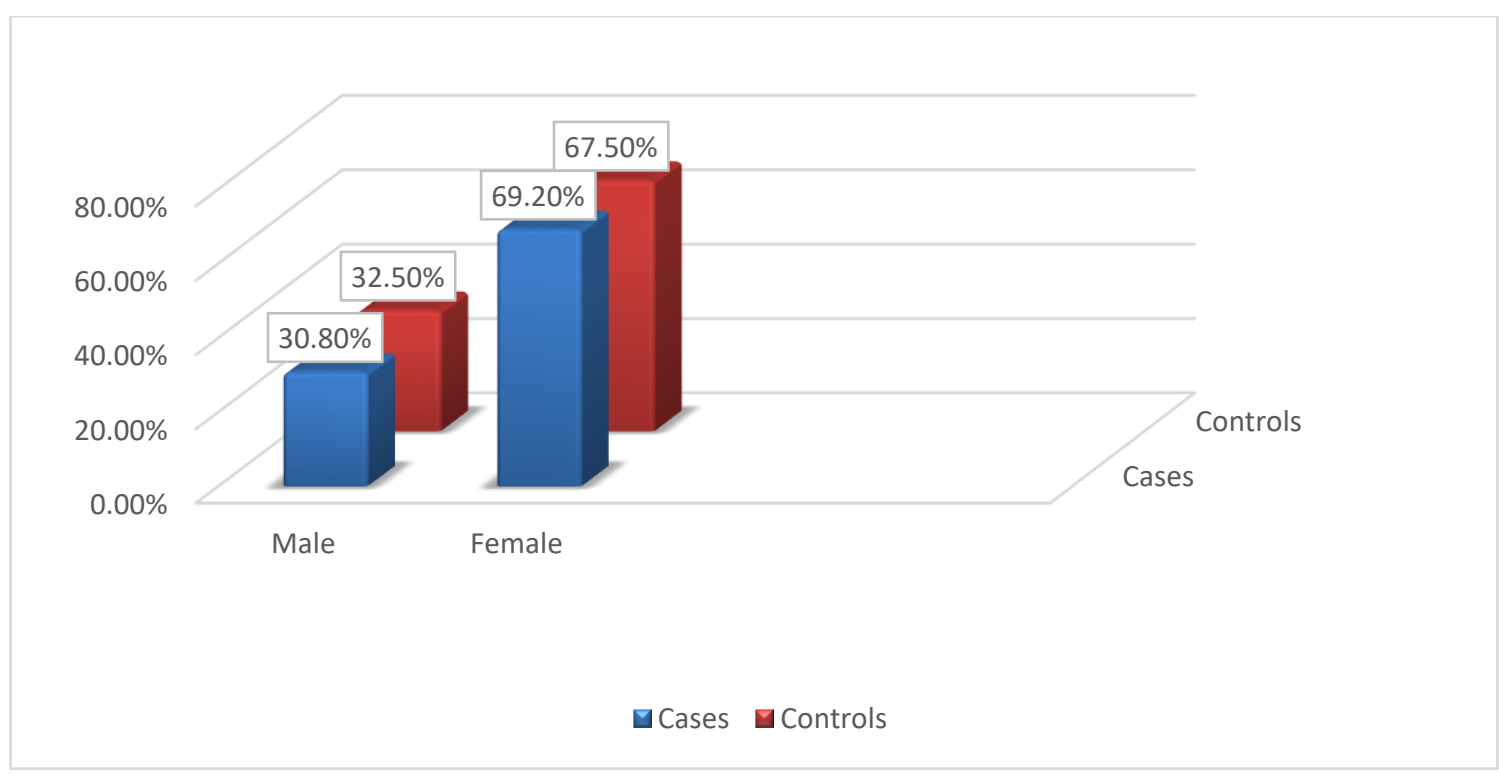

Fig (1b) Sex in patients and control group. 
SOHAG MEDICAL JOURNAL

Vol. 25 No. 2 April 2021
The Impact of Programmed Death-1 on Immune Doaa mohamed bardis

Table (1): Demographic and laboratory data of the studied groups.

\begin{tabular}{|l|l|l|l|}
\hline Parameter & Patients group & Control group & p-value \\
\hline $\begin{array}{l}\text { Age } \\
\text { Children (median) }\end{array}$ & 5 & 6 & \\
Adult (median) & 30 & 37 & 0.120 \\
\hline $\begin{array}{l}\text { Sex } \\
\text { Female }\end{array}$ Male & $67.5 \%$ & $69.2 \%$ & 0.655 \\
\hline $\begin{array}{l}\text { WBCS } \\
\text { Median }\end{array}$ & $32.5 \%$ & $30.8 \%$ & 0.884 \\
Range & 8.675 & 6.11 & \\
\hline Lymphocyte count & $3.27-21.6$ & $4.5-10.5$ & 0.001 \\
$\begin{array}{l}\text { Median } \\
\text { Range }\end{array}$ & 3.435 & 1.07 & \\
\hline $\begin{array}{l}\text { Hemoglobin } \\
\text { Median }\end{array}$ & $0.897-11.69$ & $0.996-5.1$ & 0.003 \\
Range & & & \\
\hline $\begin{array}{l}\text { PLT } \\
\text { Median }\end{array}$ & 4.61 & 4.39 & 0.105 \\
Range & $4.59-16.1$ & $8.2-14.5$ & \\
\hline $\begin{array}{l}\text { PD-1CD4 } \\
\text { Median } \\
\text { Range }\end{array}$ & 12.45 & 272 & $<0.001$ \\
\hline
\end{tabular}

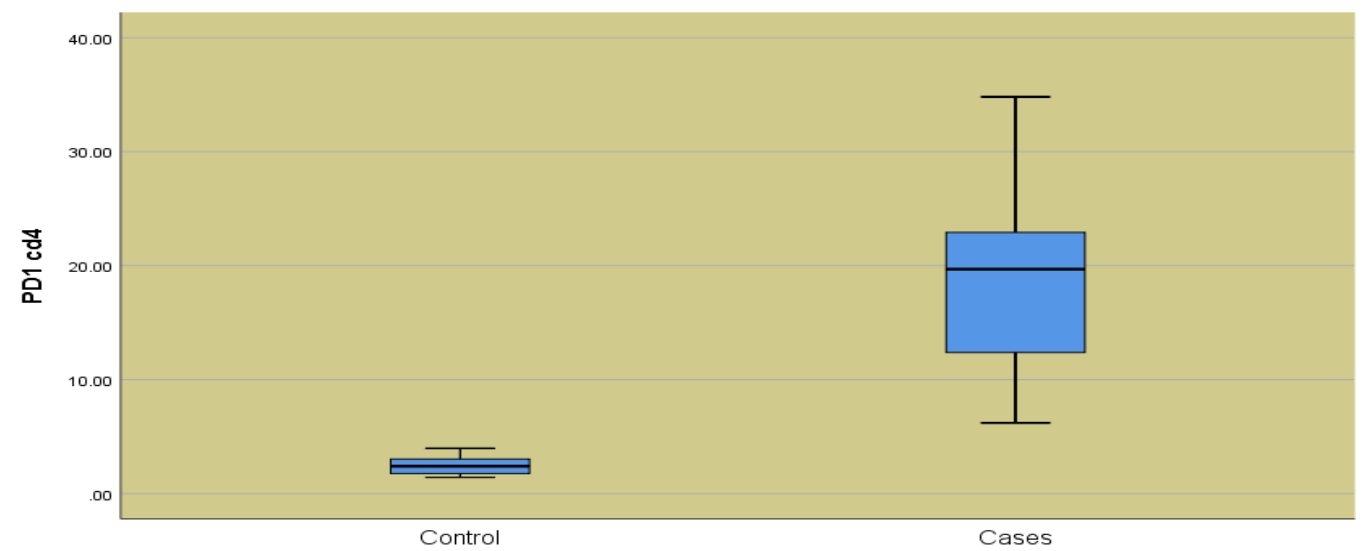

Fig (2): Comparison between case and control group regarding PD-1 expression.

(a)

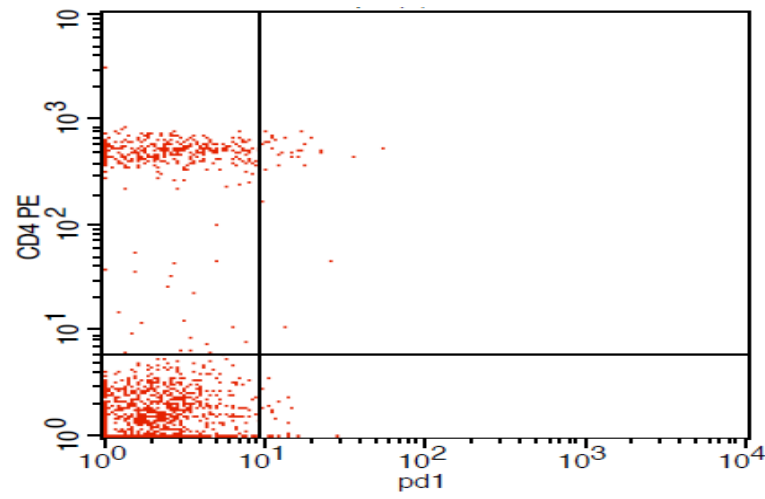

(b)

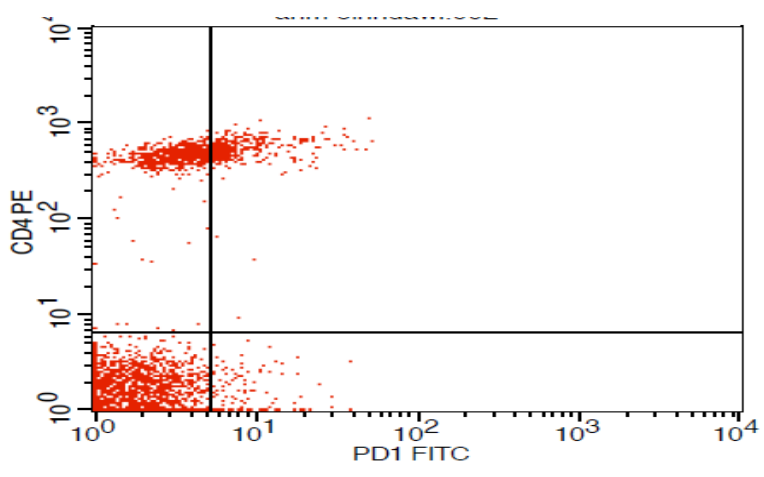

Figure (3): FCM diagram of PD-1 expression in (a) control case and (b) patient case 
SOHAG MEDICAL JOURNAL

Vol. 25 No. 2 April 2021
The Impact of Programmed Death-1 on Immune

Doaa mohamed bardis

Table (2): Clinical presentation of bleeding.

\begin{tabular}{|l|c|c|}
\hline \multicolumn{1}{|c|}{ Bleeding type } & $\begin{array}{c}\text { Count } \\
(\text { no. })\end{array}$ & $\begin{array}{c}\text { Percent } \\
(\boldsymbol{\%})\end{array}$ \\
\hline Cutaneous bleeding & 14 & $35 \%$ \\
\hline Mucocutaneous bleeding & 14 & $35 \%$ \\
\hline Mucosal bleeding & 12 & $30 \%$ \\
\hline Organ bleeding & 0 & $0 \%$ \\
\hline Total & 40 & $100 \%$ \\
\hline
\end{tabular}

Table (3): Correlations between PD-1 expression and other laboratory data.

\begin{tabular}{|l|l|l|l|l|}
\hline PD-1CD-4 & \multicolumn{2}{|l|}{ Patients group } & \multicolumn{2}{l|}{ Control group } \\
\hline & $\mathrm{r}$ & $\mathrm{p}$-value & $\mathrm{r}$ & $\mathrm{p}$-value \\
\hline Platelets & 0.337 & $0.033(\mathrm{~S}) *$ & -0.18 & $0.379(\mathrm{NS})$ \\
\hline lymphocytes & -0.144 & $0.374(\mathrm{NS})$ & -0.129 & $0.53(\mathrm{NS})$ \\
\hline MPV (fl) & -0.104 & $0.523(\mathrm{NS})$ & 0.037 & $0.857(\mathrm{~N} . S)$. \\
\hline CD-4\% & 0.13 & $0.423(\mathrm{~N} . S)$. & -0.279 & $0.167(\mathrm{~N} . S)$. \\
\hline
\end{tabular}

Table (4): ROC curve of PD-1 in predicting ITP

\begin{tabular}{|l|c|l|l|l|l|l|l|l|}
\hline P-value & PPV & Specificity & Sensitivity & CI & AUC & Cutoff & Marker \\
\hline$<0.001^{*}$ & 96.3 & 100 & 100 & 97.5 & $0.993-1$ & 0.998 & $10.27 \%$ & PD-1 \\
\hline
\end{tabular}

(ROC): Receiver operating characteristic, NPV negative predictive value, PPV positive predictive value, $\mathrm{CI}$ confidence interval, AUC area upper curve

Table 5: Odd's ratio of PD1 in prediction the degree of thrombocytopenia

\begin{tabular}{|l|l|l|l|}
\hline & \multicolumn{1}{|c|}{ Odd's ratio } & \multicolumn{2}{c|}{$95 \%$ C.I. for Odd's ratio } \\
\hline 0.187 & 3.773 & 0.525 & 27.098 \\
\hline
\end{tabular}

Table 6: Correlations between PD-1 CD4 and bleeding type in ITP patients.

\begin{tabular}{|c|c|c|c|}
\hline Parameter & $\begin{array}{c}\text { Mucocutaneous cases } \\
(\text { no. 12) }\end{array}$ & $\begin{array}{c}\text { Cutaneous cases } \\
(\text { no.28) }\end{array}$ & P-value \\
\hline $\begin{array}{c}\text { Pd-1 CD-4 \% } \\
\text { (median) }\end{array}$ & 13.655 & 20.35 & $0.05($ S. $)$ \\
\hline
\end{tabular}




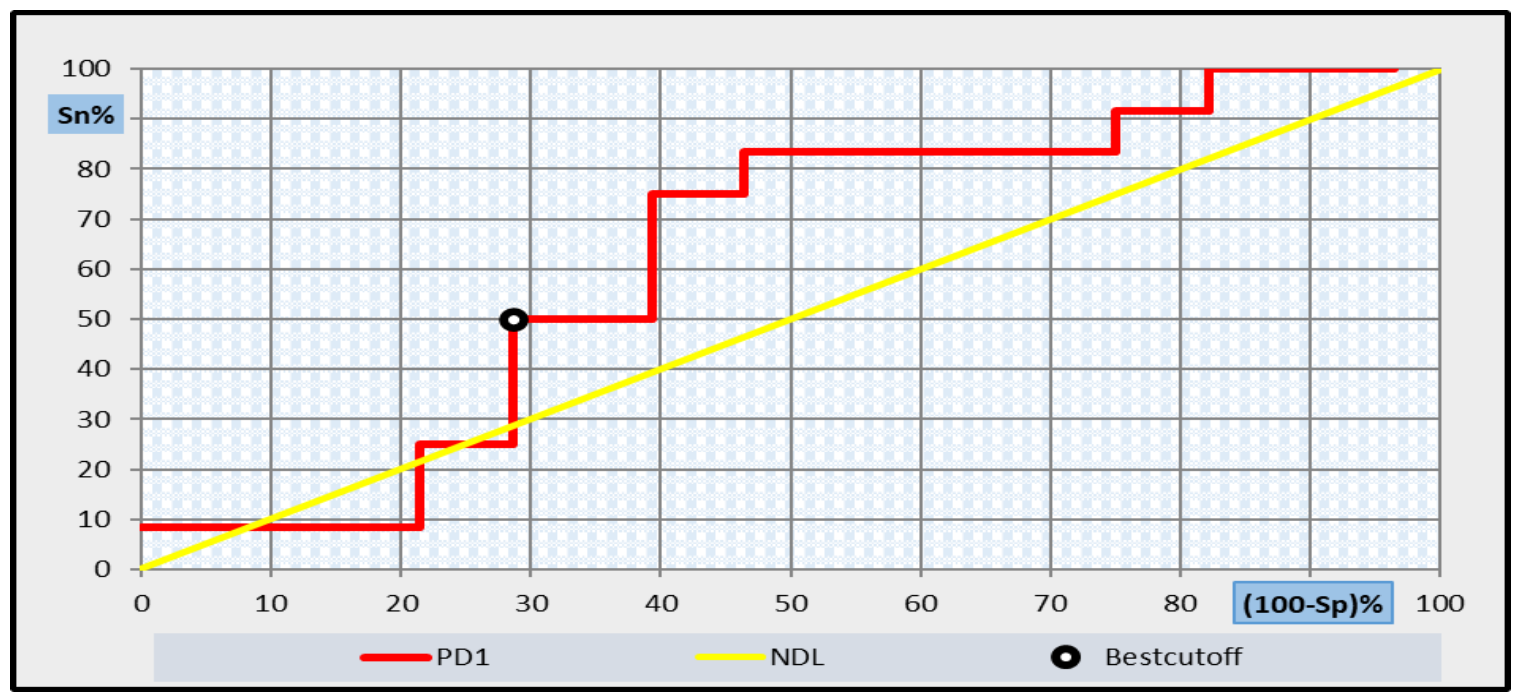

Figure (4): ROC curve of PD-1for discriminating cases with mucocutaneous bleeding than those with cutaneous bleeding.

\begin{tabular}{|l|l|c|c|c|c|}
\hline Test Result Variable(s) & Area & Std. Error & \multirow{2}{*}{ P-value } & \multicolumn{2}{|c|}{ Asymptotic 95\% Confidence Interval } \\
\cline { 5 - 6 } & & & & Lower Bound & Upper Bound \\
\hline PD-1 & 0.603 & 0.092 & $0.215(\mathrm{NS})$ & 0.196 & 0.554 \\
\hline
\end{tabular}

(ROC): Receiver operating characteristic, (Sn: sensitivity 50\%), (Sp: specificity $71.9 \%$ )

\section{Discussion:}

Immune thrombocytopenic purpura (ITP) is an acquired autoimmune bleeding disorder affecting both children and adults. patients lose immune tolerance to selfantigens on the platelet surface, which start by a process of cell-mediated and humoral-mediated destruction of their platelets ${ }^{(\mathbf{1})}$. ITP patients had enhanced activation of Th1 and Th17 cells, as well as increased cytokine release by $\mathrm{Th} 1$ and Th17 cells ${ }^{(3)}$. On the stimulated T cells, B cells, NK cells, monocytes, dendritic cells (DCs), and T regulatory cells, programmed death-1 (PD-1) is an important inhibitor of the immune system. Programmed death ligand-1 (PD-L1) and programmed death ligand-2 (PD-L2) are two ligands for PD-1. T cell proliferation and cytokine production are inhibited when the PD-1/PD-L1 signaling pathway is activated $^{(4)}$. The expression of the PD-1 negative costimulatory molecule on CD4
T cells was investigated in this work. The significance of PD-1, expression is investigated in relation to various clinical and laboratory data and controls. PD-1 CD-4 $+\mathrm{T}$-cells are increased significantly in the cases group than the control group. This finding ensures the important role of PD1 molecule in inhibiting the autoimmune process in ITP. This result with Wang et al. (2018) who discovered that the proportion of PD-1 on CD4+T cells in ITP patients' blood was considerably greater than in healthy controls. There is a significant positive correlation between PD-1 expression on CD4+T-cell and PLT. This explains the important role of the PD-1 molecule in inhibiting the immune destruction of PLT so PLT count increases with increase expression of PD-1 on autoreactive helper T-cell. Wang et al. (2018) found the same significant positive correlation between PD-1 on CD4+T cells in ITP patients and the count of the platelet. PD-1 expression significantly decreases 
in cases with mucocutaneous bleeding than in cases with cutaneous bleeding. This insure the important role of PD-1 expression in inhibiting autoreactive T-cells and so autoimmune destruction of PLT, in cases with PLT count below $20 \times 10^{9} / \mathrm{L}$ the level of PD-1 expression is lower when compared with cases with higher PLT count so it has a good prognostic value. This finding with Wong et al. (2013) who find that that the suppressive capacity of CD4(+) T(reg) depended on PD-1 expression. Zhong et al. (2016) confirm our result as they found significantly lower expression of PD-1 in chronic ITP patients and its importance in stopping the immune destruction of PLT and decrease its level make the condition chronic. PD1 has a perfect differentiating power between ITP and controls, with the most relevant cut point is $10.27 \%$, with a sensitivity of $97.5 \%$ and a specificity of $100 \%$.

\section{Conclusion:}

PD-1 expression on CD4+Tcells has a role in the pathogenesis of ITP and clinical presentation of bleeding but has no predictive value in the degree of thrombocytopenia.

\section{References:}

1. Nomura S., "Advances in diagnosis and treatments for immune thrombocytopenia." Clinical Medicine Insights: Blood Disorders. (2016); 9: 15-22

2. Iraqi M., Perdomo, J., Yan and F., Immune thrombocytopenia: antiplatelet autoantibodies inhibit proplatelet formation by megakaryocytes and impair platelet production in vitro. Hematologica. (2015); 100(5): 623-632.

3. Xie J., D. Cui, Y. Liu, J. Jin, H. Tong, L. Wang, G. Ruan, Y. Lu, and H. Yuan, "Changes in follicular helper $\mathrm{T}$ cells in idiopathic thrombocytopenic purpura patients." International journal of biological sciences. (2015); 11(2): 220.

4. Patsoukis N., Q. Wang, L. Strauss, and V. A. Boussiotis, "Revisiting the PD-1 pathway." Science Advances. (2020); 6(38): eabd2712.

5. Jiang X., Wang, J., Deng and X., Role of the tumor microenvironment in PD-L1/PD-1mediated tumor immune escape. Molecular cancer. (2019); 18(1): 10.

6. Kroenke M.A, Eto, D., Locci and M., Bcl6, and Maf cooperate to instruct human follicular helper CD4 T cell differentiation. J Immunol. (2012); 188:3734-44.

7. Nie M., Y. Liu, X.-x. Li, Y.-n. Min, D.-d. Yang, Q. Li, Q. Feng, Y. Hou, G.-s. Li and J.-z. Sun, "PD-1/PD-L pathway potentially involved in ITP immunopathogenesis." Thrombosis and hemostasis. (2019); 119(05): 758-765.

8. Xie J., D. Cui, Y. Liu, J. Jin, H. Tong, L. Wang, G. Ruan, Y. Lu, and H. Yuan, "Changes in follicular helper $\mathrm{T}$ cells in idiopathic thrombocytopenic purpura patients." International journal of biological sciences. (2015); 11(2): 220.

9. Births Atesoglu E., P. Tarkun, E. T. Demirsoy, A. Geduk, O. Mehtap, A. Batman, F. Kaya, M. B. Cekmen, Z. Gulbis, and A. Hacihanefioglu, "Soluble Programmed Death 1 (PD-1) Is Decreased in Patients with Immune Thrombocytopenia (ITP) Potential Involvement of PD-1 Pathway in ITP Immunopathogenesis." Clinical and Applied Thrombosis/Hemostasis. (2016); 22(3): 248251.

10. Wang Y., N. Pang, X. Wang, Y. Liu, X. Wang, L. Wang, and M. Sun, "Percentages of PD-1(+) CD4(+) T cells and PD-L1(+) DCs are increased and SPD-1 level is elevated in patients with immune thrombocytopenia." Hum Vaccin Immunother. (2018);14(4): 832-838.

11. Wong M., A. La Cava and B. H. Hahn, "Blockade of programmed death-1 in young (New Zealand Black x New Zealand White) 
SOHAG MEDICAL JOURNAL

Vol. 25 No. 2 April 2021

F1 mice promote the suppressive capacity of $\mathrm{CD} 4+$ regulatory $\mathrm{T}$ cells protecting from lupus-like disease." J Immunol. (2013); 190(11): 5402-5410.

12. Zhong J., S. Chen, L. Xu, J. Lai, Z. Liao,
The Impact of Programmed Death-1 on Immune Doaa mohamed bardis

T. Zhang, Z. Yu, Y. Lu, L. Yang and $X$. $\mathbf{W u}$, "Lower expression of PD-1 and PD-L1 in peripheral blood from patients with chronic ITP." Hematology. (2016); 21(9): 552-557. 